\title{
ASYMPTOTIC OSCILLATIONS OF SOLUTIONS OF SCALAR CONSERVATION LAWS WITH CONVEXITY UNDER THE ACTION OF A LINEAR EXCITATION
}

BY

\author{
ATHANASIOS N. LYBEROPOULOS
}

University of Oklahoma, Norman, Oklahoma

1. Introduction. Consider the nonlinear partial differential equation

$$
\partial_{t} u(x, t)+\partial_{x} f(u(x, t))+g(u(x, t))=0, \quad-\infty<x<\infty, 0 \leq t<\infty,
$$

where $f(\cdot)$ and $g(\cdot)$ are given smooth functions defined on $(-\infty, \infty)$ and taking values, along with the unknown $u(x, t)$, in $(-\infty, \infty)$. The above equation, which is commonly called a balance law (or conservation law, in the absence of the nonhomogeneous term $g$ ), serves as the classical prototype for systems of equations that govern the evolution of one-dimensional continuous media with "elastic" response.

Despite the apparent simplicity of (1.1), it is well known that the Cauchy problem for it does not possess globally defined smooth solutions. As a matter of fact, even if the initial data

$$
u(x, 0)=u_{0}(x), \quad-\infty<x<\infty,
$$

are $C^{\infty}$-smooth, solutions generally stay smooth only up to a critical time beyond which the nonlinear structure of $f(\cdot)$ leads to the generation of discontinuities (shocks) that tend to dominate the wave pattern thereafter. However, when $g(u)$ is either dissipative or it grows no faster than linear in $u$, then, for any bounded $u_{0}(\cdot)$ of locally bounded variation on $(-\infty, \infty)$, there exists a unique admissible global solution of (1.1), (1.2), in the class $B V$ of functions of locally bounded variation in the sense of Tonelli and Cesari $[14,10]$.

Natural questions arise concerning the large time behavior of admissible $B V-$ solutions of (1.1), (1.2). When the supply term $g(\cdot)$ vanishes a detailed description of the mechanisms that affect the asymptotic behavior of solutions, as $t \rightarrow \infty$, is available, under a variety of assumptions on the initial data $u_{0}(\cdot)$. In particular, when $u_{0}(\cdot)$ is periodic with mean zero then, if $f(\cdot)$ is convex, waves are confined and their interaction induces $O\left(t^{-1}\right)$ decay [11, 1], while, if $f(\cdot)$ has a single inflection point, then $f^{\prime}(u(x, t))$ decays as $O\left(t^{-1}\right)$ at a uniform rate, independent of the size of the data $[9,4]$.

Received January 4, 1990.

This research was completed while the author was a doctoral student in the Division of Applied Mathematics at Brown University.

(C) 1990 Brown University 
By contrast, very little is known about the large time behavior of solutions of the inhomogeneous conservation law (1.1), especially in the case where no convexity restrictions on $f(\cdot)$ are imposed [3]. In particular, it is known that if $f(\cdot)$ is uniformly convex and $u_{0}(\cdot)$ is periodic then the total variation of $u(\cdot, t)$ over a period decays to zero provided that $g(\cdot)$ is appropriately dissipative.

It is therefore of interest to discuss the situation in which there is competition between all the dissipative mechanisms that are present in the homogeneous case, $g \equiv 0$, and an excitation, for instance as in

$$
\partial_{t} u(x, t)+\partial_{x} f(u(x, t))-u(x, t)=0 .
$$

Our aim here is to study the asymptotic shape of solutions of (1.3) under continuous initial data (1.2) that are $L$-periodic and have mean zero, i.e.,

$$
\begin{gathered}
u_{0}(\cdot) \in C^{0}(-\infty, \infty) ; \quad u_{0}(x+L)=u_{0}(x), \quad L>0, \\
\int_{0}^{L} u_{0}(x) d x=0 .
\end{gathered}
$$

We consider $f(u) \in C^{2}(-\infty, \infty)$ which is strictly (but not necessarily uniformly) convex, i.e., $f^{\prime \prime}(\cdot)$ is nonnegative and does not vanish identically on any interval, normalized so that

$$
f(0)=f^{\prime}(0)=0, \quad u f^{\prime}(u)>0, \quad u \neq 0 .
$$

A typical example is provided by the equation

$$
\partial_{t} u(x, t)+\partial_{x}\left(|u(x, t)|^{p}\right)-u(x, t)=0, \quad p \geq 2 .
$$

From the perspective of large-time behavior of solutions, one is naturally tempted to explore the implications of the group-invariance of (1.3) under the translation group

$$
(x, t, u) \mapsto(x+c \tau, t+\tau, u), \quad \tau \in \mathbb{R},
$$

generated by $\partial_{t}+c \partial_{x}$, where $c$ is a fixed constant. Global invariants of this group are

$$
\eta=x-c t, \quad v=u
$$

so that a group-invariant solution $v=\varphi(\eta)$ takes the familiar form $u(x, t)=$ $\varphi(x-c t)$ which determines a travelling wave moving at constant speed $c$. Imposing the restriction on $\varphi(\cdot)$ to be periodic and of class $B V$ we find that (1.3) admits a family of periodic travelling-wave solutions which, in fact, are stationary. It becomes therefore conceivable that, as a result of the competition between the dissipative flux-term $f_{x}$ and the exciting source-term $-u$, the solution no longer decays but, instead, approaches an asymptotically sustained oscillation. Indeed, as our analysis shows, admissible weak solutions of (1.3) under periodic initial data with mean zero converge, as $t \rightarrow \infty$, to a particular member of the above family of stationary waves.

Though still awaiting, it seems that an exhaustive study of the asymptotic behavior of solutions of the model equation (1.1) could provide the necessary technical 
background for exploring similar questions for systems of hyperbolic balance laws $[7,12,6]$.

The fundamental tool in our investigation will be the concept of a generalized characteristic in the sense of [2]. A characteristic of (1.1), associated with a classical solution $u(x, t)$ is defined as a trajectory of the ordinary differential equation

$$
\frac{d x}{d t}=f^{\prime}(u(x, t))
$$

For weak solutions the same definition is adopted, but now (1.8) is interpreted as a contingent equation in the sense of Filippov [8]. As a consequence of the Filippov theory, through any point $(\bar{x}, \bar{t})$ of the upper half-plane passes at least one forward characteristic, defined on a right maximal interval $[\bar{t}, \tau), \tau \leq \infty$, and at least one backward characteristic defined on a left maximal interval $(\sigma, \bar{t}], \sigma \geq 0$. The set of forward (or backward) characteristics through $(\bar{x}, \bar{t})$ spans the funnel confined between a minimal and a maximal forward (or backward) characteristic through $(\bar{x}, \bar{t})$. Of course, these extremal forward (or backward) characteristics need not be distinct. It turns out that all generalized characteristics propagate with either classical characteristic speed or with shock speed, but it is the special properties of the extremal backward ones, that play the key role in the analysis.

It is well worth noting here that an investigation concerning the asymptotic behavior of admissible weak solutions of (1.3) under periodic initial data with mean zero, when $f(\cdot)$ is not convex but has just one inflection point, has also been carried out by the author [13], for the model equation

$$
\partial_{t} u(x, t)-\partial_{x}\left(|u(x, t)|^{p} u(x, t)\right)-u(x, t)=0, \quad p>1,
$$

whose behavior is to be contrasted to that of (1.7).

2. Generalized characteristics. We consider an admissible solution of (1.3), that is, a measurable function $u(x, t)$ which satisfies (1.3) in the sense of distributions and for all $x \in(-\infty, \infty)$ and all $t \in[0, \infty)$ the one-sided limits $u(x+, t), u(x-, t)$ exist and

$$
u(x+, t) \leq u(x-, t) .
$$

For a thorough discussion on the motivation of the admissibility condition (2.1) and other analogous conditions see [5]. Normalizing $u(x, t)$ we assume further that it is continuous from the left, i.e.,

$$
u(x, t)=u(x-, t), \quad-\infty<x<\infty, 0 \leq t<\infty .
$$

A generalized characteristic of (1.3), associated with a solution $u(x, t)$, is a Lipschitz trajectory $\xi(\cdot):[a, b] \rightarrow(-\infty, \infty), 0 \leq a<b<\infty$, of the ordinary differential equation (1.8) in the sense of Filippov [8], i.e.,

$$
\dot{\xi}(t) \in\left[f^{\prime}(u(\xi(t)+, t)), f^{\prime}(u(\xi(t), t))\right],
$$

for almost all $t \in[a, b]$. 
LemMA 2.1. Let $\xi(\cdot):[a, b] \rightarrow(-\infty, \infty)$ and $\zeta(\cdot):[a, b] \rightarrow(-\infty, \infty), 0 \leq a<$ $b<\infty$, be Lipschitz continuous curves. Then, for almost all $\sigma, \tau, a \leq \sigma<\tau \leq b$,

$$
\begin{aligned}
\int_{\xi(\tau)}^{\zeta(\tau)} & u(x, \tau) d x-\int_{\xi(\sigma)}^{\zeta(\sigma)} u(x, \sigma) d x-\int_{\sigma}^{\tau} \int_{\xi(t)}^{\zeta(t)} u(x, t) d x d t \\
= & \int_{\sigma}^{\tau}\{f(u(\xi(t), t))-\dot{\xi}(t) u(\xi(t), t)\} d t \\
& -\int_{\sigma}^{\tau}\{f(u(\zeta(t)+, t))-\dot{\zeta}(t) u(\zeta(t)+, t)\} d t .
\end{aligned}
$$

Proof. See Dafermos [2].

Setting $\xi(t) \equiv \zeta(t)$ in (2.3) we deduce the following corollary which shows that generalized characteristics must propagate with either classical characteristic speed or with shock speed.

COROllary 2.1. Let $\xi(\cdot):[a, b] \rightarrow(-\infty, \infty)$ be a generalized characteristic. Then for almost all $t \in[a, b]$

$$
\dot{\xi}(t)= \begin{cases}f^{\prime}(u(\xi(t), t)) & \text { if } u(\xi(t)+, t)=u(\xi(t), t), \\ \frac{f(u(\xi(t)+, t))-f(u(\xi(t), t))}{u(\xi(t)+, t)-u(\xi(t), t)} & \text { if } u(\xi(t)+, t)<u(\xi(t), t) .\end{cases}
$$

A characteristic $\xi(\cdot)$ on $[a, b]$ is called genuine if $u(\xi(t)+, t)=u(\xi(t), t)$ for almost all $t \in[a, b]$ while a characteristic $\chi(\cdot)$ defined on a maximal interval $[\bar{t}, \sigma)$ is called a shock if $u(\chi(t)+, t)<u(\chi(t), t)$ for all $t \in(\bar{t}, \sigma)$.

In view of the next theorem, genuine characteristics are classical characteristics.

THEOREM 2.1. Let $\xi(\cdot)$ be a genuine characteristic on $[a, b]$. Then there is a function $v(\cdot)$ on $[a, b]$ so that $(\xi(\cdot), v(\cdot))$ is a continuously differentiable solution of the classical characteristic equations

$$
\left.\begin{array}{l}
\dot{\xi}(t)=f^{\prime}(v(t)) \\
\dot{v}(t)=v(t) .
\end{array}\right\}
$$

Furthermore, for any $t \in(a, b)$

$$
u(\xi(t), t)=v(t)=u(\xi(t)+, t)
$$

while

$$
\begin{gathered}
u(\xi(a), a) \leq v(a) \leq u(\xi(a)+, a), \\
u(\xi(b), b) \geq v(b) \geq u(\xi(b)+, b) .
\end{gathered}
$$

Proof. See Dafermos [2].

According to the theory of contingent equations of type (2.2), [8], through any point $(\bar{x}, \bar{t})$ of the upper half-plane, passes a funnel of backward (forward) generalized characteristics, confined between a minimal and a maximal one. In Theorems $2.2,2.3$, and 2.4 we exhibit the remarkable properties of these extremal backward and forward characteristics (for the proofs see [2]). 
THEOREM 2.2. The minimal and maximal backward characteristics $\zeta_{-}(t ; \bar{x}, \bar{t})$ and $\zeta_{+}(t ; \bar{x}, \bar{t})$ through any fixed point $(\bar{x}, \bar{t}) \in(-\infty, \infty) \times(0, \infty)$ are genuine characteristics determined by solving (2.5) with initial conditions $(\xi(\bar{t}), v(\bar{t}))=$ $(\bar{x}, u(\bar{x}, \bar{t}))$ and $(\xi(\bar{t}), v(\bar{t}))=(\bar{x}, u(\bar{x}+, \bar{t}))$, respectively. Therefore, $\zeta_{-}(t ; \bar{x}, \bar{t})$ and $\zeta_{+}(t ; \bar{x}, \bar{t})$ coincide if and only if $u(\bar{x}+, \bar{t})=u(\bar{x}, \bar{t})$. Furthermore, they are both defined on $[0, \bar{t}]$.

THEOREM 2.3. The minimal and maximal forward characteristics through any fixed point $(\bar{x}, \bar{t}) \in(-\infty, \infty) \times(0, \infty)$ always coincide into a single characteristic $\chi(t ; \bar{x}, \bar{t})$ which is defined on $[\bar{t}, \infty)$.

THEOREM 2.4. Let $\chi(\cdot)$ denote the unique forward characteristic issuing from a point $(\bar{x}, \bar{t}) \in(-\infty, \infty) \times(0, \infty)$ with $u(\bar{x}+, \bar{t})<u(\bar{x}, \bar{t})$. Then $\chi(\cdot)$ is a shock defined on $[\bar{t}, \infty)$. The functions $u(\chi(t), t), u(\chi(t)+, t)$ are continuous from the right on $[\bar{t}, \infty), \chi(\cdot)$ is differentiable from the right on $[\bar{t}, \infty)$, and

$$
\frac{d^{+}}{d t} \chi(t)=\frac{f(u(\chi(t)+, t))-f(u(\chi(t), t))}{u(\chi(t)+, t)-u(\chi(t), t)}, \quad t \in[\bar{t}, \infty),
$$

while for any $\bar{s} \in(\bar{t}, \infty)$

$$
\left\{\begin{array}{l}
\lim _{t \uparrow \bar{s}} u(\chi(t), t) \leq u(\chi(\bar{s}), \bar{s}), \\
\lim _{t \uparrow^{\bar{s}}} u(\chi(t)+, t) \geq u(\chi(\bar{s})+, \bar{s}) .
\end{array}\right.
$$

Furthermore, if $\bar{s}$ is a point of continuity of the functions $u(\chi(t), t), u(\chi(t)+, t)$, then $(\chi(\bar{s}), \bar{s})$ is a point of continuity of $u(x, t)$ relative to the set $\mathscr{L}_{-}=\{(x, t)$ : $x<\chi(t)\}$, the limit being $u(\chi(\bar{s}), \bar{s})$, and also relative to the set $\mathscr{L}_{+}=\{(x, t)$ : $x>\chi(t)\}$ with limit $u(\chi(\bar{s})+, \bar{s})$.

3. Travelling-wave solutions. Our objective here is to determine whether the balance law (1.3), with $f(u)$ satisfying (1.6), admits special, $L$-periodic weak solutions $(L>0)$ which are travelling waves with constant speed of propagation $c$, i.e., $u(x, t)=\varphi(x-c t), c \in \mathbb{R}$.

Let us introduce the variable $\eta=x-c t$. In view of our earlier discussion we shall seek such solutions in the class of $B V$-functions. Accordingly, $\varphi(\cdot)$ may have an at most countable set of discontinuities over $(-\infty, \infty)$ at which, of course, the admissibility criterion (2.1) must be satisfied. We restrict ourselves, however, to the case where $\varphi(\cdot)$ is left-continuous and has over any period only a finite number of discontinuities (jumps).

THEOREM 3.1. The only possible weak solutions of $(1.3)$ (where $f(u)$ is strictly convex and satisfies (1.6)) that are travelling waves with constant speed of propagation $c, L$-periodic, of locally bounded variation, left-continuous, and have over a period $[a, a+L], a \in \mathbb{R}$, only a finite number of jump-discontinuities, say at the points $a \leq \eta_{1}<\eta_{2}<\cdots<\eta_{k} \leq a+L$, are the stationary ones $(c=0)$, i.e., of the form $u(x, t)=\varphi(x)$. Furthermore, if $\varphi(\cdot) \not \equiv 0$ then it is necessarily discontinuous on $[a, a+L]$ while it is a continuous solution (in the sense of distributions) of the 
ordinary differential equation

$$
\frac{d f(\varphi(\eta))}{d \eta}-\varphi(\eta)=0
$$

in any interval $\left(\eta_{i}, \eta_{i+1}\right), i=1,2, \ldots, k-1$, between two adjacent discontinuities and for $i=1,2, \ldots, k$,

$$
\begin{gathered}
f\left(\varphi\left(\eta_{i}+\right)\right)=f\left(\varphi\left(\eta_{i}\right)\right), \\
\varphi\left(\eta_{i}+\right)<0<\varphi\left(\eta_{i}\right) .
\end{gathered}
$$

Proof. In view of (1.3), (2.1), it is clear that $\varphi(\cdot)$ must be a continuous solution of the ordinary differential equation

$$
c \frac{d \varphi(\eta)}{d \eta}-\frac{d f(\varphi(\eta))}{d \eta}+\varphi(\eta)=0
$$

in any open subinterval $\left(\eta_{i}, \eta_{i+1}\right), i=1,2, \ldots, k-1$, while at each point $\eta_{i}$, the admissibility condition

$$
\varphi\left(\eta_{i}+\right)<\varphi\left(\eta_{i}\right),
$$

and the Rankine-Hugoniot jump condition

$$
f\left(\varphi\left(\eta_{i}+\right)\right)-f\left(\varphi\left(\eta_{i}\right)\right)=c\left(\varphi\left(\eta_{i}+\right)-\varphi\left(\eta_{i}\right)\right)
$$

must be satisfied. Note first that by the mean-value theorem, (3.5) and (3.6) imply the familiar entropy inequality

$$
f^{\prime}\left(\varphi\left(\eta_{i}+\right)\right)<c<f^{\prime}\left(\varphi\left(\eta_{i}\right)\right), \quad i=1,2, \ldots, k .
$$

We claim that for such nontrivial solutions to exist, it is necessary that $c=0$. Indeed, suppose $c>0$ (the case $c<0$ can be treated along similar lines). Rewriting Eq. (3.4) in the form

$$
\frac{d \varphi}{d \eta}=\frac{\varphi}{f^{\prime}(\varphi)-c}
$$

it is easily seen that, unless $\varphi(\cdot) \equiv 0$, the speed $c$ must take values in the range of $f^{\prime}(\cdot)$ and $\varphi(\cdot)$ must be discontinuous on $[a, a+L]$, because otherwise, it would be impossible to satisfy, by virtue of (3.7), the periodicity requirement

$$
\varphi(a)=\varphi(a+L), \quad a \in \mathbb{R} .
$$

Therefore, without loss of generality, we can assume that $\eta_{1}=a, \eta_{k}=a+L$. Consider now the restriction of $\varphi(\cdot)$ over the interval $\left(\eta_{1}, \eta_{2}\right)$ and assume that it is not identically zero there. In view of (3.7), (3.8), and the properties of $f(\cdot)$ we see that if $f^{\prime}\left(\varphi\left(\eta_{1}+\right)\right)>0$ then $\varphi(\cdot)$ is decreasing on $\left(\eta_{1}, \eta_{2}\right)$ and $\varphi\left(\eta_{1}+\right)>\varphi\left(\eta_{2}\right) \geq 0$ so that $f^{\prime}\left(\varphi\left(\eta_{2}\right)\right)<c$ which violates (3.7). On the other hand if $f^{\prime}\left(\varphi\left(\eta_{1}+\right)\right)<0$ then $\varphi(\cdot)$ is increasing on $\left(\eta_{1}, \eta_{2}\right)$ and $\varphi\left(\eta_{1}+\right)<\varphi\left(\eta_{2}\right) \leq 0$ so that $f^{\prime}\left(\varphi\left(\eta_{2}\right)\right) \leq 0<c$ which again violates (3.7). Thus the claim is proved. Setting $c=0$ in (3.4), (3.6), and (3.7) we obtain (3.1), (3.2), and (3.3), respectively. The proof is complete.

On account of $(3.1),(3.3)$, we observe that the restriction of $\varphi(\cdot)$ over any subinterval $\left(\eta_{i}, \eta_{i+1}\right)$ is an increasing function that changes from negative to positive sign in a continuous fashion. In fact, $\varphi(\cdot)$ may vanish identically on intervals $\left[\lambda_{i}, \mu_{i}\right]$, 
where $\eta_{i}<\lambda_{i} \leq \mu_{i}<\eta_{i+1}$ for all $i$. Finally, integrating (3.1) over a period and using (3.9) we also conclude that

$$
\int_{a}^{a+L} \varphi(\eta) d \eta=0
$$

In the special case of Eq. (1.7), we have the following

THEOREM 3.2. All nontrivial weak solutions of the balance law (1.7) that are travelling waves with constant speed of propagation $c, L$-periodic, of locally bounded variation, left-continuous and have over a period $[a, a+L], a \in \mathbb{R}$, only a finite number of jump-discontinuities, say at the points $a=\eta_{1}<\eta_{2}<\cdots<\eta_{k}=a+L$, are in fact stationary, i.e., of the form $u(x, t)=\varphi(x)$. Furthermore, in any interval $\left(\eta_{i}, \eta_{i+1}\right], i=1,2, \ldots, k-1$,

$$
\varphi(x)=\left\{\begin{array}{cl}
-\left\{\varphi^{p-1}\left(\eta_{i}\right)-\frac{p-1}{p}\left(x-\eta_{i}\right)\right\}^{\frac{1}{p-1}}, & \eta_{i}<x \leq \lambda_{i} \\
0, & \lambda_{i} \leq x \leq \mu_{i} \\
\left\{\varphi^{p-1}\left(\eta_{i+1}\right)+\frac{p-1}{p}\left(x-\eta_{i+1}\right)\right\}^{\frac{1}{p-1}}, & \mu_{i} \leq x \leq \eta_{i+1}
\end{array}\right.
$$

where $\lambda_{i}=\eta_{i}+\frac{p}{p-1} \varphi^{p-1}\left(\eta_{i}\right)$ and $\mu_{i}=\eta_{i+1}-\frac{p}{p-1} \varphi^{p-1}\left(\eta_{i+1}\right)$.

A straight-forward calculation shows that relations (3.11) in conjunction with the periodicity requirement (3.9) impose restrictions on the range of $\varphi(\cdot)$, namely for all $x \in(-\infty, \infty)$

$$
0 \leq|\varphi(x)| \leq\left(\frac{p-1}{2 p} L\right)^{\frac{1}{p-1}} .
$$

On the other hand, it is easily seen that there are infinitely many functions $\varphi(\cdot)$ that satisfy the requirements of Theorem 3.2, depending on the number and strength of jump-discontinuities one wishes to allow over a period.

4. Asymptotic behavior of solutions. Suppose $u(x, t)$ is an admissible $B V$ solution of $(1.3),(1.2)$ where the initial data $u_{0}(\cdot)$ satisfy $(1.4),(1.5)$. It is clear that, for each fixed $t \in[0, \infty), u(\cdot, t)$ will also be $L$-periodic with mean zero.

Let $[\bar{x}, \bar{x}+L]$ be a fixed period-interval and for every $t \in[0, \infty)$ define the set

$$
\mathscr{Z}_{t} \equiv\{x \in[\bar{x}, \bar{x}+L]: u(x+, t)=u(x, t)=0\} .
$$

LemMA 4.1. For any $t \in[0, \infty), \mathscr{Z}_{t}$ is nonempty and compact. Furthermore, $\mathscr{Z}_{t} \subseteq \mathscr{Z}_{s}$ for any $0 \leq s \leq t$.

Proof. On account of $(1.4),(1.5)$ it is clear that $\mathscr{Z}_{0} \neq \varnothing$. Suppose now that $\mathscr{Z}_{\bar{t}}=\varnothing$ for some $\bar{t}>0$. If $0 \leq u(\bar{x}+, \bar{t})$ and $u(\bar{x}, \bar{t})>0$ then, since $u(\cdot, \bar{t})$ has zero mean over a period, there must be a point $y \in(\bar{x}, \bar{x}+L)$ such that $u(y+, \bar{t})=$ $u(y, \bar{t})<0$, which, in view of the admissibility condition (2.1), would imply that $u(\bar{x}, \bar{t})=u(\bar{x}+L, \bar{t}) \leq 0$. On the other hand, if $u(\bar{x}+, \bar{t})<0 \leq u(\bar{x}, \bar{t})$ or $u(\bar{x}+, \bar{t}) \leq u(\bar{x}, \bar{t})<0$, then, again because of $(2.1), u(\cdot, \bar{t})$ would have negative mean over $(\bar{x}, \bar{x}+L]$. Hence in all possible cases we reach a contradiction which shows that $\mathscr{Z}_{t} \neq \varnothing$ for all $t \in[0, \infty)$. To show that $\mathscr{Z}_{t}$ is also compact take any decreasing (increasing) sequence $\left\{x_{k}\right\}_{k=1}^{\infty} \in \mathscr{Z}_{t}$ such that $x_{k} \rightarrow x^{+}\left(x_{k} \rightarrow x^{-}\right)$. 
Clearly, $x \in[\bar{x}, \bar{x}+L]$ while $u(x+, t)=\lim _{x_{k} \rightarrow x^{+}} u\left(x_{k}, t\right)=0 \quad(u(x, t)=$ $\left.\lim _{x_{k} \rightarrow x^{-}} u\left(x_{k}, t\right)=0\right)$ for any $t \in[0, \infty)$. Thus $u(\cdot, t)$ is continuous at $x$ and equal to zero. Finally, take a point $x \in \mathscr{Z}_{t}$ and consider the unique backward characteristic $\zeta(s ; x, t) \equiv \zeta_{ \pm}(s ; x, t)$ through $(x, t)$. By Theorems 2.1, 2.2, we know that $\zeta(s ; x, t) \equiv x$ and $u(\zeta(s ; x, t) \pm, t) \equiv u(x \pm, t)=0$ for all $s \in[0, t]$. Therefore $x \in \mathscr{Z}_{s}$ for all $s \in[0, t]$ and the proof is complete.

From Lemma 4.1 we deduce immediately the following

Corollary 4.1. Let $\left\{t_{m}\right\}_{m=1}^{\infty}$ be any positive sequence such that $t_{m} \rightarrow \infty$. Then the sequence $\left\{\bigcap_{k=1}^{m} \mathscr{Z}_{t_{k}}\right\}_{m=1}^{\infty}$ converges, as $m \rightarrow \infty$, to a set $\mathscr{Z}_{\infty}$ which is nonempty, compact, and independent of $\left\{t_{m}\right\}_{m=1}^{\infty}$.

THeOrem 4.1. Let $u(x, t)$ be an admissible $B V$ solution of (1.3), (1.2) where the initial data $u_{0}(\cdot)$ satisfy $(1.4),(1.5)$ and $f(u)$ is strictly convex and conforms with (1.6). Suppose $\bar{x}$ is such that $u(\bar{x}, t)=u(\bar{x}+, t)=0$ for all $t \in[0, \infty)$, (see Corollary 4.1). Then the interval $[\bar{x}, \bar{x}+L]$ is decomposed into two, uniquely defined, disjoint subsets $\mathscr{Z}_{\infty}$ and $\mathscr{G}_{\infty}$ with the properties:

i) $\mathscr{Z}_{\infty}$ is nonempty and compact. Also, $u(x, t)=u(x+, t)=0$ for all $x \in \mathscr{Z}_{\infty}$ and all $t \in[0, \infty)$.

ii) $\mathscr{G}_{\infty}=\varnothing$ if and only if $u_{0}(x) \equiv 0$ on $(-\infty, \infty)$.

iii) If $\mathscr{G}_{\infty}$ is nonempty then it is an (at most) countable union of pairwise disjoint open intervals, $\mathscr{G}_{\infty}=\bigcup_{k}\left(a_{k}, b_{k}\right), a_{k} \in \mathscr{Z}_{\infty}, b_{k} \in \mathscr{Z}_{\infty}$, and as $t \rightarrow \infty$,

$\int_{u(x \pm, t) e^{-t}}^{u(x \pm, t)} \quad w^{-1} f^{\prime}(w) d w= \begin{cases}x-a_{k}+o(1), & a_{k} \leq x<\chi_{k}(t) \\ x-b_{k}+o(1), & \chi_{k}(t)<x \leq b_{k}\end{cases}$

where $\chi_{k}(\cdot)$ is a shock.

Furthermore,

$$
\int_{a_{k}}^{b_{k}} u(x, t) d x=\int_{a_{k}}^{b_{k}} u_{0}(x) d x=0 \text { for all } t \in[0, \infty) .
$$

Proof. Clearly, without loss of generality, we can modify definition (4.1) so that $\bar{x} \in \mathscr{Z}_{\infty}$ (as in Corollary 4.1). Then, by the periodicity of $u(\cdot, t)$, we must also have $\bar{x}+L \in \mathscr{Z}_{\infty}$. Assume now that $u_{0}(\cdot) \not \equiv 0$ on $[\bar{x}, \bar{x}+L]$, i.e., $\mathscr{Z}_{0}^{c} \neq \varnothing$, and consider the set

$$
\mathscr{G}_{\infty} \equiv[\bar{x}, \bar{x}+L] \backslash \mathcal{Z}_{\infty}
$$

By Corollary 4.1 we deduce that $\mathscr{G}_{\infty}$ is nonempty, open, and bounded. Therefore, it can be uniquely represented in the form of an, at most, countable union of pairwise disjoint open intervals, the end points of which do not belong to the set $\mathscr{G}_{\infty}$, i.e.,

$$
\mathscr{G}_{\infty}=\bigcup_{k}\left(a_{k}, b_{k}\right), \quad a_{k} \in Z_{\infty}, b_{k} \in Z_{\infty} .
$$

Now fix $k \geq 1$, take an arbitrary point $x \in\left(a_{k}, b_{k}\right)$ and consider the two extremal backward characteristics $\zeta_{ \pm}(s ; x, t)$ through $(x, t)$ with $t>0$. On account of 
Theorems 2.1 and 2.2 we have

$$
\begin{aligned}
& u(x \pm, t)=u_{0}\left(\zeta_{ \pm}(0 ; x, t)\right) e^{t}, \\
& x-\zeta_{ \pm}(0 ; x, t)=\int_{0}^{t} f^{\prime}\left(u\left(\zeta_{ \pm}(s ; x, t), s\right)\right) d s \\
&=\int_{0}^{t} f^{\prime}\left(u(x \pm, t) e^{s-t}\right) d s \\
&=\int_{u(x \pm, t) e^{-t}}^{u(x \pm, t)} w^{-1} f^{\prime}(w) d w, \\
&\left|x-\zeta_{ \pm}(0 ; x, t)\right| \leq b_{k}-a_{k}<L,
\end{aligned}
$$

for all $t \in(0, \infty)$, and so, in view of $(1.6)$, there must be $\theta \in\left[a_{k}, b_{k}\right]$ such that

$$
u_{0}\left(\zeta_{ \pm}(0 ; x, t)\right) \underset{t \rightarrow \infty}{\longrightarrow} u_{0}(\theta)=0
$$

We claim that, as $t \rightarrow \infty$, the interceptors $\zeta_{ \pm}(0 ; x, t)$ must accumulate at $a_{k}$ or $b_{k}$. Indeed, suppose there is a sequence $\left\{\zeta_{ \pm}\left(0 ; x_{m}, t_{m}\right)\right\}_{m=1}^{\infty}, a_{k}<x_{m}<b_{k}$, which converges, as $t_{m} \rightarrow \infty$, to a point $\bar{\theta} \in\left(a_{k}, b_{k}\right)$ such that $u_{0}(\bar{\theta})=0$. Then, by the theory of contingent equations of type (2.2), [8], the sequence of backward characteristics $\left\{\zeta_{ \pm}\left(s ; x_{m}, t_{m}\right)\right\}_{m=1}^{\infty}$ should converge to a genuine characteristic $\xi(\cdot)$ through $(\bar{\theta}, 0)$, defined on $[0, \infty)$ and confined in the strip formed by the two parallel forward characteristics through the points $\left(a_{k}, 0\right)$ and $\left(b_{k}, 0\right)$. Therefore, by (4.6), (4.7), (4.8), and Theorem 2.1 we should also have that

$$
\begin{gathered}
u(\xi(s)+, s)=u(\xi(s), s)=u_{0}(\xi(0))=u_{0}(\bar{\theta})=0, \\
\xi(s)=\xi(0)=\bar{\theta},
\end{gathered}
$$

for all $s \in[0, \infty)$, which implies that $\bar{\theta} \in \mathscr{Z}_{\infty}$, contrary to our assumption.

Consequently, for large $t$ there is $\chi_{k}(t)$ with the property that, if $a_{k} \leq x<\chi_{k}(t)$, then $\zeta_{ \pm}(0 ; x, t)$ are near $a_{k}$ while, if $\chi_{k}(t)<x \leq b_{k}$ then $\zeta_{ \pm}(0 ; x, t)$ are near $b_{k}$ and so from (4.7) we obtain the asymptotic result (4.2). It is also clear that for large $t, \chi_{k}(t)$ is a shock. In order to show (4.3) we apply Lemma 2.1 for the backward characteristics through the points $\left(a_{k}, t\right)$ and $\left(b_{k}, t\right), t>0$, and we obtain

$$
\int_{a_{k}}^{b_{k}} u(x, t) d x=e^{t} \int_{a_{k}}^{b_{k}} u_{0}(x) d x \text { for all } t \in[0, \infty),
$$

which implies that the integral on the right-hand side should vanish because, otherwise, there would be $x \in\left(a_{k}, b_{k}\right)$ such that $\lim _{t \rightarrow \infty}|u(x, t)|=\infty$ in contradiction to (4.2). The proof is complete.

Note that the asymptotic location of the shock $\chi_{k}(\cdot)$ can be determined on combining (4.2) with (4.3). In particular, for the special case of Eq. (1.7) the asymptotic 
relation (4.2) takes the form

$$
u(x \pm, t)= \begin{cases}\left\{\frac{(p-1)\left(x-a_{k}\right)}{p\left(1-e^{-(p-1) t}\right)}\right\}^{\frac{1}{p-1}}+o(1), & a_{k} \leq x<\chi_{k}(t) \\ -\left\{\frac{(p-1)\left(b_{k}-x\right)}{p\left(1-e^{-(p-1) t}\right)}\right\}^{\frac{1}{p-1}}+o(1), & \chi_{k}(t)<x \leq b_{k}\end{cases}
$$

where

$$
\chi_{k}(t)=\frac{1}{2}\left(a_{k}+b_{k}\right)+o(1)
$$

When $u_{0}(\cdot)$ changes sign a finite number of times over a period, it is very easy to infer that the decomposition (4.5) of the set $\mathscr{G}_{\infty}$ contains actually only a finite number of pairwise disjoint open intervals $\left(a_{k}, b_{k}\right), k=1,2, \ldots, \ell$, and so, on account of Theorem 3.1 , one readily concludes that in this case $u(x, t)$ approaches asymptotically a specific travelling-wave solution $\varphi(x)$. In general, however, $u(x, t)$ approaches, as $t \rightarrow \infty$, a unique function $\varphi_{\infty}(x)$ which can have over a period an, at most, countable number of discontinuities and may be considered as the limit of some converging sequence $\left\{\varphi_{n}(x)\right\}_{n=1}^{\infty}$ of travelling-wave solutions each of which satisfies the requirements of Theorem 3.1 .

Acknowledgment. I would like to thank Professor Constantine M. Dafermos who suggested this problem and provided many fruitful discussions during the preparation of this work.

\section{REFERENCES}

[1] C. M. Dafermos, Characteristics in hyperbolic conservation law', Nonlinear Analysis and Mechanics (R.J. Knops, Ed.), Research Notes in Math. No. 17, Pitman, London, 1977, pp. 1-58

[2] C. M. Dafermos, Generalized characteristics and the structure of solutions of hyperbolic conservation laws, Indiana Univ. Math. J. 26, 1097-1119 (1977)

[3] C. M. Dafermos, Asymptotic behavior of solutions of hyperbolic balance law's, Bifurcation Phenomena in Mathematical Physics (C. Bardos and D. Bessis. Eds.), D. Reidel, Dordrecht, 1979, pp. $521-533$

[4] C. M. Dafermos, Regularity and large time behavior of solutions of a conservation law without convexity, Proc. Roy. Soc. Edinburgh 99A, 201-239 (1985)

[5] C. M. Dafermos, Hyperbolic systems of conservation law's, Systems of Nonlinear Partial Differential Equations (J.M. Ball, Ed.), NATO ASI Series C No. 111, D. Reidel, Dordrecht, 1983, pp. 25-70

[6] C. M. Dafermos, Generalized characteristics in hyperbolic systems of conservation law's, Arch. Rational Mech. Anal. 107, 127-155 (1989)

[7] R. J. Diperna, Decay and asymptotic behavior of solutions to nonlinear hyperbolic systems of conservation laws, Indiana Univ. Math. J. 24, 1047-1071 (1975)

[8] A. F. Filippov, Differential equations with discontinuous right-hand side, Mat. Sbornik (N.S.) 51, 99-128 (1960) (English translation: Amer. Math. Soc. Transl. Ser. 2 42, 199-231 (1964))

[9] J. M. Greenberg and D. D. Tong, Decay of periodic solutions of $\partial u / \partial t+\partial f(u) / \partial x=0$, J. Math. Anal. Appl. 43, 56-71 (1973)

[10] S. N. Kružkov, First order quasilinear equations in several independent variables, Mat. Sbornik (N.S.) 81, 228-255 (1970) (English translation: Math. USSR-Sbornik 10, 217-243 (1970))

[11] P. D. Lax, Hyperbolic systems of conservation law's II, Comm. Pure Appl. Math. 10, 537-566 (1957) 
[12] T.-P. Liu, Decay to $N$-waves of solutions of general systems of nonlinear hyperbolic conservation laws, Comm. Pure Appl. Math. 30, 585-610 (1977)

[13] A. N. Lyberopoulos, Asymptotic oscillations of solutions of scalar conservation laws with or without convexity under the action of a linear excitation, Ph.D. dissertation, Brown University, 1989

[14] A. I. Vol'pert, The space BV and quasilinear equations, Mat. Sbornik 73 (1967) (English translation: Math. USSR-Sbornik 2, 225-267 (1967)) 